\title{
Probing biological light-harvesting phenomena by optical cavities
}

\author{
Filippo Caruso, ${ }^{1}$ Semion K. Saikin, ${ }^{2,3}$ Enrique Solano, ${ }^{4,5}$ Susana F. Huelga, ${ }^{1}$ Alán Aspuru-Guzik, ${ }^{2}$ and Martin B. Plenio ${ }^{1}$ \\ ${ }^{1}$ Institut für Theoretische Physik, Universität Ulm, Albert-Einstein-Allee 11, D-89069 Ulm, Germany \\ ${ }^{2}$ Department of Chemistry and Chemical Biology, Harvard University, 12 Oxford Street, Cambridge, MA 02138, USA \\ ${ }^{3}$ Department of Physics, Kazan Federal University, 18 Kremlyovskaya Street, Kazan 420008, Russian Federation \\ ${ }^{4}$ Departamento de Química Física, Universidad del País Vasco - Euskal Herriko Unibertsitatea, E-48080 Bilbao, Spain \\ ${ }^{5}$ IKERBASQUE, Basque Foundation for Science, Alameda Urquijo 36, 48011 Bilbao, Spain
}

\begin{abstract}
We propose a driven optical cavity quantum electrodynamics (QED) set up aimed at directly probing energy transport dynamics in photosynthetic biomolecules. We show that detailed information concerning energy transfer paths and delocalization of exciton states can be inferred (and exciton energies estimated) from the statistical properties of the emitted photons. This approach provides us with a novel spectroscopic tool for the interrogation of biological systems in terms of quantum optical phenomena which have been usually studied for atomic or solid-state systems, e.g. trapped atoms and semiconductor quantum dots.
\end{abstract}

Introduction.- Following a series of fascinating nonlinear optical experiments with light-harvesting complexes (LHCs) involved in natural photosynthesis [1-5], the analysis of exciton dynamics in biological systems has attracted a rapidly growing interest from different scientific communities. It has been demonstrated that the essentially lossless excitation energy transfer (EET) in these structures does not follow the weak-coupling Förster theory [6] where the excitation energy incoherently hops between single molecules. Indeed, exciton states in LHCs are frequently delocalized over several molecules. Several theoretical studies have analyzed EET in such pigment-protein complexes and demonstrated that an intricate interplay between environmental noise (dephasing) and quantum coherence is crucial to explain the remarkably high EET efficiency (above $95 \%$ ) from the peripheral antenna, collecting external light, to a reaction center, where electronic energy is transformed into chemical form [7-17].

On the other hand, the ability to control light-matter interaction for single atoms [18] or quantum dots [19] in high-quality optical cavities, e.g. photonic crystals, micropillars, and microdisk resonators, has recently allowed the theoretical and experimental analysis of fundamental quantum phenomena arising at a single photon level [20]. In particular, quantum optical phenomena such as the vacuum Rabi splitting, singleatom lasing, photon squeezing, anti-bunching, and matterlight entanglement have been explored in the strong-coupling limit, i.e. when the exchange energy rate between the cavity mode and the trapped system is larger than the involved decay processes [21, 22]. In this regime, the cavity and trapped system can no longer be described as separate subsystems, i.e. there is a splitting between energy eigenstates into different manifolds (dressed states or polaritons associated to the Jaynes-Cummings model). Polariton physics has had a recent surge in interest in the context of coupled cavity arrays [23[25] and in Bose-Einstein condensation of exciton polaritons [26, 27]. A different, yet related, lasing process [28] was also observed in organic molecular crystals in cavities [29].

Here, we present the extension of the rich physics of cavity quantum electrodynamics to the investigation of the internal excitation energy transfer dynamics of bio-molecules. In particular, we propose theoretically an experimental scheme in

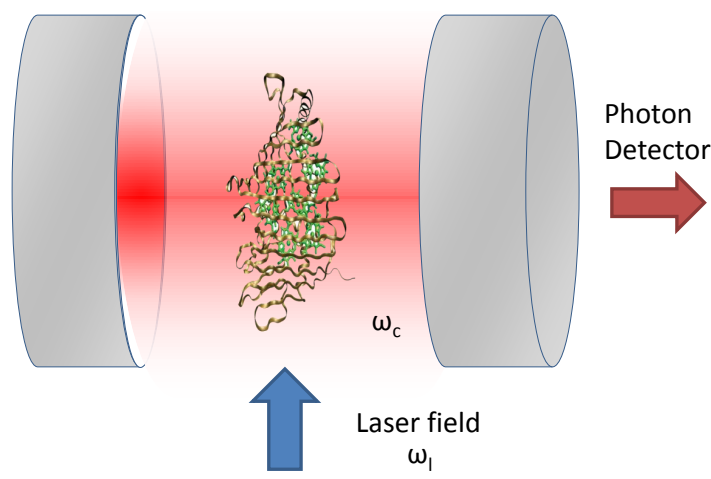

FIG. 1: Artistic impression of the proposed setup: a sample of lightharvesting complexes is confined in an optical cavity (resonance frequency $\omega_{c}$ ) with a quality factor $Q$. The sample is subjected to an orthogonal driving laser field of frequency $\omega_{l}$. Photons leaking out of the cavity are collected by a photon detector.

which a sample of LHCs, driven by an external laser field, is trapped inside an optical cavity. Proof-of-principle demonstrations for solution-phase cavity-enhanced spectroscopy were reported in [30], where absorption measurements with Bacteriochlorophyll a (BChla) in solution were performed. In this Letter, the main goal is to detect quantum phenomena associated with the emitted quantized light [31], in order to probe biological coherence in photosynthetic complexes, in a different and complementary way with respect to 2D nonlinear electronic spectroscopy used so far in Refs. [1-4, 36].

We show that the LHC exciton structure and energy transfer paths can be mapped onto the statistical properties of cavity photons such as mean photon number and second-order correlation functions and later measured through a light mode leaking out of the cavity. Furthermore, we quantify the amount of quantum correlations between the cavity mode and the biological system and discuss the possibility of polariton formation for a single LHC in a cavity.

Model.- In order to probe the exciton structure of biological systems, we essentially propose a pump-probe scheme in which the probe field is substituted by the cavity mode. The sample (system) is assumed to be confined inside an optical 
cavity with resonance frequency $\omega_{c}$, while energy is injected into the system via an external laser field (pump) with frequency $\omega_{l}$. While the pump field can be pulsed or continuous wave $(\mathrm{CW})$, the probe field (cavity mode) is always $\mathrm{CW}$. We will consider the case where the pump field is turned on at a certain time, which allows us to explore both the stationary response to the $\mathrm{CW}$ field as well as the transient excitation dynamics. Moreover, for the sake of simplicity, we consider a collinear polarization of the laser field and the cavity mode. The extension of the model to a pulsed excitation and crossfield polarizations is straightforward. The LHC exciton dynamics is characterized by monitoring the mean photon number and the second order coherence function $g^{(2)}(0)$ of the light leaking out of the cavity (probe). The coherent interaction between the cavity mode and the LHC chromophores can be written as

$$
H_{c}=\sum_{i} g \mu_{i}\left(\sigma_{i}^{+} a+\sigma_{i}^{-} a^{\dagger}\right)
$$

where $g$ is the coupling constant, $\mu_{i}$ is the projection of the transition dipole moment of a single chromophore to the polarization of the cavity mode, $\hat{\sigma}_{i}^{ \pm}$are rising and lower operators for chromophore excitations, and $\hat{a}^{\dagger}, \hat{a}$ denote the cavity photon creation/annihilation operators. See the supplementary information (SI) for additional details concerning the model. The rotating wave approximation utilized in Eq. (1) is valid for the coupling regime we consider here. For the sake of simplicity we analyze photon statistics in a single polarization mode in the cavity. In general, there are two degenerate polarization modes. However, the interaction between them is mediated by the LHC and the associated effects in the photon statistics are of higher order in the LHC-cavity coupling as compared to the single photon emission by the LHC. The coupling coefficient $g$ is given by $g=\sqrt{\frac{\omega_{c}}{2 \varepsilon V}}$, where $\hbar=1, V$ is the mode volume, and $\varepsilon$ is the permittivity of the medium between the cavity mirrors. In the following, we assume $\varepsilon=2$, which is approximately the dielectric constant of a solvent at optical frequencies. The cavity properties can be described by the quality factor $Q$ and the mode volume $V$. While the former parameter determines the cavity photon escape rate as $\kappa=\omega_{c} / Q$, the latter one controls the coupling with the LHC. Experimentally achievable values of the quality factor can be as large as $Q=10^{5}-10^{7}[20,32,33]$. The fundamental limit of the mode volume is determined by the photon wavelength $V_{f}=(\lambda / 2)^{3}$ and is dependent on the dielectric constant of the medium. While the mode volume in photonic crystal cavities is about $\lambda^{3}$ [20], to the best of our knowledge the state-of-the-art mode volume, demonstrated for a FabryPerot cavity design [33], which can be used for molecular spectroscopy, is $V_{\exp } \approx 5.5 \lambda^{3}$. This value corresponds to $g \approx 0.015 \mathrm{~cm}^{-1} / \mathrm{D}$ coupling between the LHC exciton and the cavity vacuum state for $\lambda_{\text {vacuum }}=800 \mathrm{~nm}$. A more optimistic estimation for the coupling constant obtained for the fundamental limit of the mode volume, i.e. $V_{f} \approx 0.023 \mu \mathrm{m}^{3}$, is $g_{\mathrm{th}} \approx 0.1 \mathrm{~cm}^{-1} / D$. In the following, we analyze the results corresponding to the latter case in order to enhance the fundamental issues of molecular spectroscopy in a cavity we want to stress, while we also discuss (in SI) the state-of-the-art case to answer the question of which effects can be observed at the present stage of technological advances. As an example of an LHC, we choose the Fenna-Matthews-Olson (FMO) pigment-protein complex involved in the natural photosynthesis of green-sulphur bacteria. The FMO subunit has seven [34] strongly coupled bacteriochlorophyll (BChl) molecules (sites) [1, 2]. The Hamiltonian of the complex can be written as

$$
H_{s}=\sum_{j} \omega_{j} \sigma_{j}^{+} \sigma_{j}^{-}+\sum_{j \neq l} v_{j, l}\left(\sigma_{j}^{-} \sigma_{l}^{+}+\sigma_{j}^{+} \sigma_{l}^{-}\right),
$$

where only lowest electronic transitions of the molecules are taken into account, $\omega_{j}$ is the site energy, and $v_{j, l}$ denotes the coherent coupling between the corresponding sites. The single exciton transitions in the FMO complex are estimated to be in the range of $12150-12750 \mathrm{~cm}^{-1}$ [35, 36]. The excitation dynamics in the FMO-cavity system can be described by a general Liouville-von Neumann equation for the density matrix

$$
\dot{\rho}=-i[H, \rho]+\mathcal{L}(\rho),
$$

where the total Hamiltonian $H$ includes the FMO Hamiltonian Eq. 22, the cavity Hamiltonian $H_{\mathrm{ph}}=\omega_{c} a^{\dagger} a$, the interactions between FMO and the cavity Eq. (1), and also the coupling between the FMO and an external field $E\left(\omega_{l}, t\right)$ given by

$$
H_{l}(t)=-\sum_{i} \mu_{i} E\left(\omega_{l}, t\right) \sigma_{i}^{+}+h . c .
$$

The dephasing and relaxation channels in the isolated FMO system impose an irreversible dynamics of an initially created exciton state. The simplest way to introduce these noise processes into the dynamics is using a Lindblad form for the term $\mathcal{L}(\rho)$ where the environmental noise is described by the site energy fluctuations (phenomenological Haken-Strobl noise) [37] with dephasing noise strength $\gamma$. This analysis will bring out the basic principles of our proposal. Extensions to non-Markovian effects which can arise from strong coupling and/or the form of the environment spectral function $[11,12,38,-41]$ as well as other cavity setups involving additional laser fields, the role of multiple excitations, and other generalizations will be investigated in a forthcoming paper.

Results and discussion.- In Fig. 2, we show the mean photon number in the cavity, in the stationary state, as a function of the driving field and the cavity mode frequencies together with the electron excitation spectrum of FMO. This 2D map is obtained $45 \mathrm{ps}$ after a strong laser driving field of intensity $I \approx 110 \mathrm{KW} / \mathrm{cm}^{2}$ has been turned on. The estimated values of electronic transition dipoles of the FMO chromophores are in the range of $3-14 \mathrm{D}$ (see SI for the details of the model). Thus, the maximal coupling energy between the driving field and the excitons is of the order of $1-7 \mathrm{~cm}^{-1}=30-210 \mathrm{GHz}$ and the exciton-cavity coupling is about 5 times smaller. The cavity photon population saturates to a stationary regime on 20 ps timescale showing 


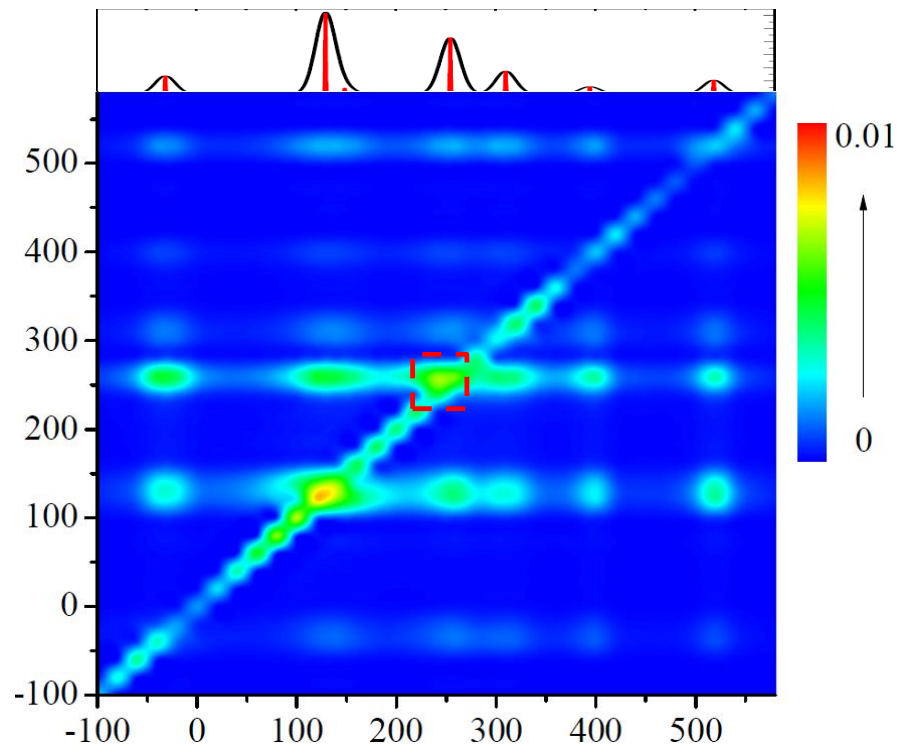

FIG. 2: Stationary mean photon number of the cavity mode as a function of the cavity $\left(Q=10^{4}\right)$ resonance frequency $\omega_{c}$ (vertical axis) and the pump laser frequency $\omega_{l}$ (horizontal), both in units of $\mathrm{cm}^{-1}$, but shifted by $12195 \mathrm{~cm}^{-1} \sim 820 \mathrm{~nm}$, in the case of dephasing rate $\gamma=10 \mathrm{~cm}^{-1}$. The diagonal peaks correspond to the FMO exciton energies, while the off-diagonal peaks are related to energy transfer between different excitons. The asymmetry of the 2D spectrum, with respect to the main diagonal $\left(\omega_{l}=\omega_{c}\right)$, is due to the different strengths of the laser and the cavity fields. Top Inset: computed spectrum of electronic excitations the FMO complex. The peaks corresponding to the FMO exciton energies are broadened by the $\gamma=10 \mathrm{~cm}^{-1}$ linewidth. The red box is analyzed in Fig. 3 .

a set of peaks that are clearly associated with the exciton frequencies of the FMO complex. Indeed, in analogy to 2D spectroscopy [1-4, 36], diagonal peaks are in correspondence of the FMO exciton energies, and off-diagonal features appear due to energy transfer between different exciton states. The diagonal line in the spectra is due to both a coherent and an incoherent transfer of the photons from the laser field into the cavity. The former process is similar to the Raman scattering and goes through coherence pathways without generating an exciton population in LHC, while the latter process can be considered as a light absorption by the LHC with the following spontaneous emission of a same-frequency photon into the cavity. The horizontal lines in the spectra (off-diagonal peaks) are due to population transfer between different excitons. Weaker optical fields would result in a similar set of peaks, but the stationary regime would be achieved on a longer timescale. For the particular case shown in Fig. 22, the noise strength is $\gamma=10 \mathrm{~cm}^{-1} \sim 2 \mathrm{ps}^{-1}$ [3, 42]. Qualitatively, this picture remains valid for the values of $\gamma$ within the range $1-100 \mathrm{~cm}^{-1}$ - see SI for more data. In all our simulations, we include an isotropic averaging of the measured quantities over completely random orientations of FMOs in the cavity. In presence of an oriented sample these quantum features would be further enhanced. Stronger, albeit (biological)-structure preserving, laser driving fields,

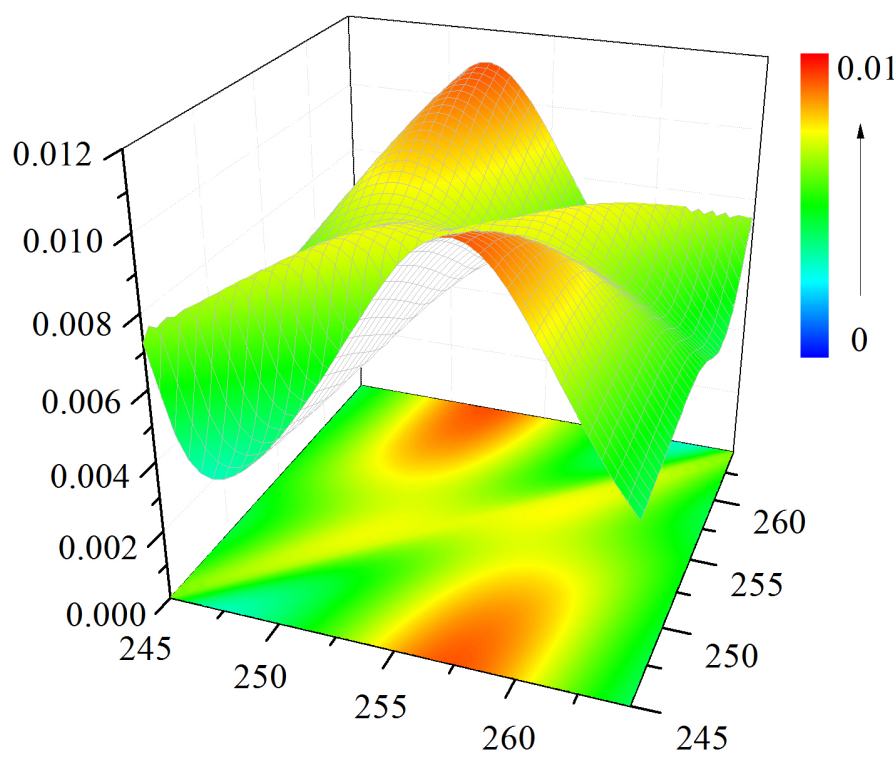

FIG. 3: Mean photon number of the stationary cavity mode as a function of the cavity $\left(Q=10^{4}\right)$ resonance frequency $\omega_{c}$ (bottom axis) and the pump laser frequency $\omega_{l}$ (right axis), both in units of $\mathrm{cm}^{-1}$ - but shifted by $12195 \mathrm{~cm}^{-1} \sim 820 \mathrm{~nm}$ - in the finely tuned range $[245,265] \mathrm{cm}^{-1}$ (red box in Fig. 2). One can clearly observe an anti-crossing effect.

can result in a fine structure of a diagonal peak. This effect is similar to a level anti-crossing or a hole burning - see Fig. 3 . Indeed, the relatively strong coupling with the pump laser field leads to a splitting of the exciton level into two dressed states corresponding to two new peaks in the mean photon number 2D map. In order to characterize quantum properties of the generated cavity photon state in its stationary state, we compute the second-order photon coherence function [43]

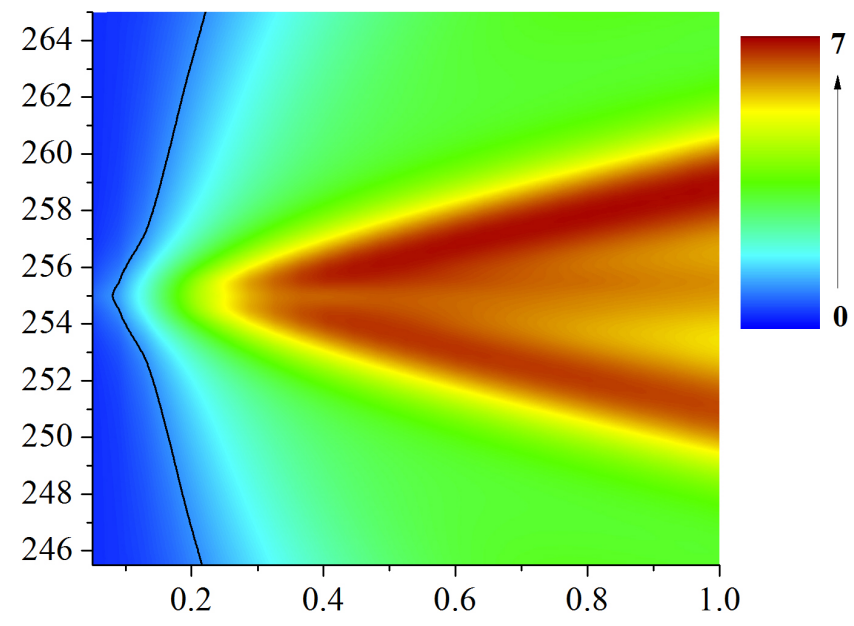

FIG. 4: Second-order coherence function at time delay zero $g^{(2)}(0)$ of the stationary cavity mode as a function of the laser field coupling (horizontal axis) and the cavity $\left(Q=10^{4}\right)$ resonance frequency $\omega_{c}$ (vertical) - in units $\mathrm{cm}^{-1}$ - for pump laser frequency $\omega_{l}=-\omega_{c}+$ $510 \mathrm{~cm}^{-1}$. The black line corresponds to $g^{(2)}(0)=1$. 


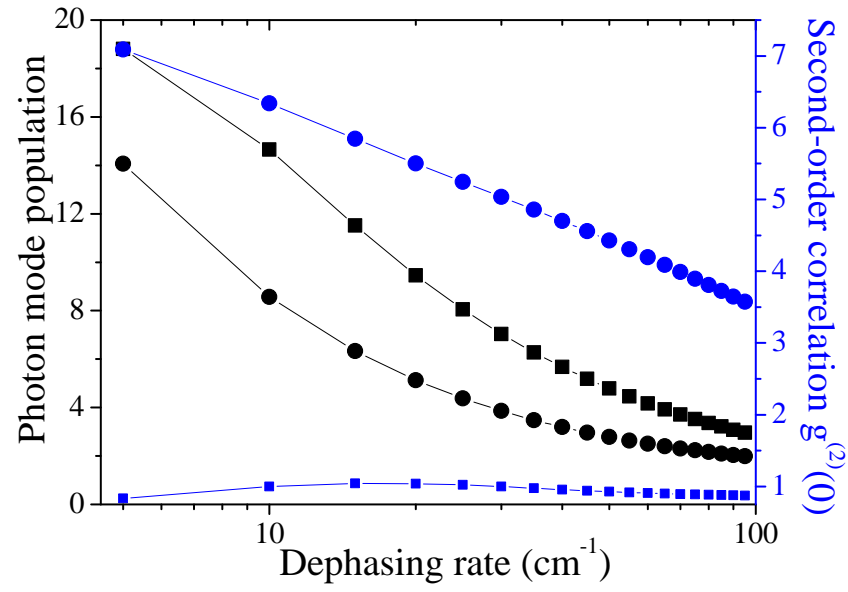

FIG. 5: Photon mode population (rescaled by a factor $10^{-3}$ ) and second-order coherence function at zero time delay $g^{(2)}(0)$ of the stationary cavity mode as a function of the dephasing rate $\left(\mathrm{cm}^{-1}\right)$, for a diagonal (circles) and off-diagonal (square) peak in the mean photon number 2D map in Fig. 2

at zero time delay $g^{(2)}(0)=\frac{\left\langle a^{\dagger} a^{\dagger} a a\right\rangle}{\left\langle a^{\dagger} a\right\rangle^{2}}-$ see Fig. 14. In general, we obtain a non-classical photon state generation $\left(g^{(2)}(0)<1\right)$ for most of the laser and cavity frequencies except of the diagonal line with $\omega_{c}=\omega_{l}$ where a coherent state $\left(g^{(2)}(0)=1\right)$ and a thermal state $\left(g^{(2)}(0)>1\right)$ can be observed. More data on $g^{(2)}(0)$ in different regimes can be found in SI. Moreover, it turns out, as in Fig. 5, that this quantity $g^{(2)}(0)$ depends on the amount of dephasing noise in the dynamics and this might become a tool to estimate the strength of interaction between the biological molecule and the external environment. Notice also that the photon mode population is more sensitive to the dephasing noise, as compared to $g^{(2)}(0)$. In addition, the new scheme we are proposing allows us also to generated non-classical states of light by means of the coherent exciton dynamics. Finally, as possible signature of polariton formation, we quantify the amount of quantum correlations between the FMO sample and the cavity mode, as measured by the logarithmic negativity [44]. It turns out that this quantity reaches a maximum at about 800 fs and around the main two diagonal peaks in the 2D map in Fig. 2- see Fig. 6 Note that the lifetime of these correlations is around $2 \mathrm{ps}^{-1}$, which is the time scale of the fastest decay process due to the presence of dephasing noise. Therefore, the coupling between the quantum cavity and the confined sample leads to the creation of non-classical correlations between these two systems. A different scheme towards entangling two FMO samples (entangled polaritons) in separate cavities is also discussed in SI.

Conclusions and outlook.- We have extended the rich physics of cavity quantum electrodynamics to biological molecules, in particular light-harvesting complexes involved in natural photosynthesis. As an example, we have considered a sample of the Fenna-Matthews- Olson (FMO) pigment-

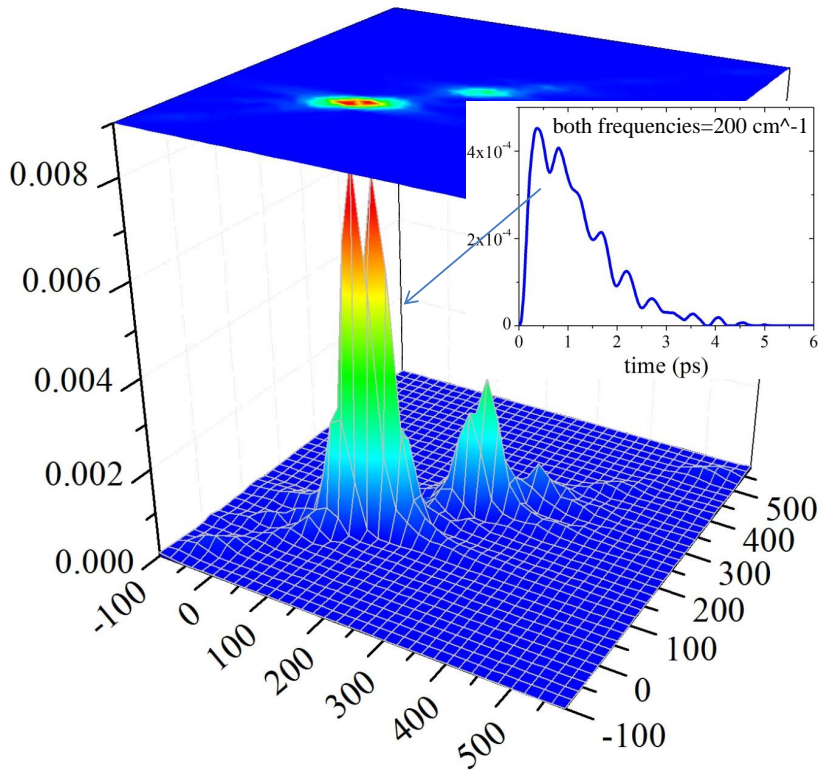

FIG. 6: Measure of quantum correlation (log-negativity) between the cavity mode and the FMO sample after $\sim 800 \mathrm{fs}$, as a function of the cavity $\left(Q=10^{4}\right)$ resonance frequency $\omega_{c}$ and the pump laser frequency $\omega_{l}$, both in units of $\mathrm{cm}^{-1}$, but shifted by $12195 \mathrm{~cm}^{-1}$, in the case of dephasing rate $\gamma=10 \mathrm{~cm}^{-1}$. Inset: Time evolution of the log-negativity measure for $\omega_{c}=\omega_{l}=200 \mathrm{~cm}^{-1}$.

protein complex inside an optical cavity and driven transversally by a laser field. Our main result is that the emission spectrum from the coupled FMO-cavity system reflects coherent energy transfer into the cavity through delocalized exciton states, as well as exciton dynamics within the FMO complex. Moreover, we have found that a strong laser field driving the exciton dynamics in the protein complex can burn a hole in the emission spectrum resulting in an additional structure of resonance peaks. Finally, the generation of quantum states of light inside the cavity, due to the interaction with the confined biological complexes, was also observed. We believe that this novel spectroscopic tool can efficiently probe both static and dynamical (system-environment) properties of natural and artificial photosynthetic structures and provide additional experimental support for the coherent dynamics unveiled by nonlinear spectroscopy experiments. This combined evidence is expected to shed further light towards the elucidation of the role that quantum coherence may play in the remarkably robust energy transport phenomena involved in natural photosynthesis.

This work was supported by DARPA-QUBE, DTRA Contract No HDTRA1-10-1-0046, the EU projects Q-ESSENCE AQUTE, CORNER, HIP, SOLID, and CCQED, the Spanish Ministry of Education (project MICINN FIS2009-12773C02-01), the Basque government (project IT472-10), the SFB/TRR21, the EU Marie-Curie Programme, and the Alexander von Humboldt Foundation. 
[1] V.I. Prokhorenko, A.R. Holzwarth, F.R. Nowak, and T.J. Aartsma, J. Phys. Chem. B 106, 9923 (2002).

[2] G.S. Engel, T.R. Calhoun, E.L. Read, T.-K. Ahn, T. Manal, Y.-C. Cheng, R.E. Blankenship, and G.R. Fleming, Nature 446, 782 (2007); H. Lee, Y-C. Cheng, and G.R. Fleming, Science 316, 1462 (2007).

[3] G. Panitchayangkoon, D. Hayes, K.A. Fransted, J.R. Caram, E. Harel, J. Wen, R.E. Blankenship, and G.S. Engel, Proc. Natl. Acad. Sci. 107, 12766 (2010).

[4] E. Collini, C.Y. Wong, K.E. Wilk, P.M.G. Curmi, P. Brumer, and G.D. Scholes, Nature 463, 644648 (2010).

[5] I.P. Mercer, Y.C. El-Taha, N. Kajumba, J.P. Marangos, J.W.G. Tisch, M. Gabrielsen, R.J. Cogdell, E. Springate, and E. Turcu, Phys. Rev. Lett. 102, 057402 (2009).

[6] T. Förster, Delocalized excitation and excitation transfer., in Modern Quantum Chemistry, Sinanoglu, O. (ed.), pages 93-137 (New York and London: Academic Press, 1965).

[7] M. Mohseni, P. Rebentrost, S. Lloyd, and A. Aspuru-Guzik, J. Chem. Phys. 129, 174106 (2008).

[8] M.B. Plenio and S.F. Huelga, New J. Phys. 10, 113019 (2008).

[9] P. Rebentrost, M. Mohseni, I. Kassal, S. Lloyd, and A. AspuruGuzik, New J. Phys. 11, 033003 (2009).

[10] A. Olaya-Castro, C.F. Lee, F. Fassioli Olsen, and N.F. Johnson, Phys. Rev. B 78, 085115 (2008).

[11] F. Caruso, A.W. Chin, A. Datta, S.F. Huelga, and M.B. Plenio, J. Chem. Phys. 131, 105106 (2009); Phys. Rev. A 81, 062346 (2010).

[12] A.W. Chin, A. Datta, F. Caruso, S.F. Huelga, and M.B. Plenio, New J. Phys. 12, 065002 (2010).

[13] F. Caruso, S.F. Huelga, and M.B. Plenio, Phys. Rev. Lett. 105, 190501 (2010).

[14] S. Shim, P. Rebentrost, S. Valleau, and A. Aspuru-Guzik, Eprint arXiv:1104.2943 (2011).

[15] J. Yuen-Zhou, M. Mohseni, J.J. Krich, and A. AspuruGuzik, Proc. Natl. Acad. Sci. USA, in press (2011), Eprint arXiv: 1006.4866

[16] P. Rebentrost and A. Aspuru-Guzik, J. Chem. Phys. 134, 101103 (2011).

[17] J. Zhu, S. Kais, P. Rebentrost, and A. Aspuru-Guzik, J. Phys. Chem. B 115, 1531-1537 (2011)

[18] H. Mabuchi and A. C. Doherty, Science 298, 1372 (2002).

[19] F. P. Laussy, E. del Valle, M. Schrapp, A. Laucht, J. J. Finley, Eprint arXiv:1104.3564(2011).

[20] G. Khitrova, H.M. Gibbs, M. Kira, S.W. Koch, and A. Scherer, Nat. Physics 2, 81-90 (2006).

[21] A. Majumdar, A. Papageorge, E.D. Kim, M. Bajscy, H. Kim, P. Petro, and Jelena Vučković, Eprint arXiv:1103.2716 (2011); A. Majumdar, M. Bajscy, and J. Vučković, Eprint arXiv:1106.1926 (2011).

[22] A. Ourjoumtsev, A. Kubanek, M. Koch, C. Sames, P. W. H. Pinkse, G. Rempe, and K. Murr, Nature 474, 623 (2011).

[23] M.J. Hartmann, F.G.S.L. Brandão, and M.B. Plenio, Nat. Phys. 2, 849 (2006).

[24] D.G. Angelakis, M.F. Santos, and S. Bose, Phys. Rev. A 76, 031805(R) (2007).

[25] A.D. Greentree, C. Tahan, J.H. Cole, and L.C.L. Hollenberg, Nat. Phys. 2, 856 (2006).

[26] J. Kasprzak et al., Nature 443, 409 (2006).

[27] H. Deng and Y. Yamamoto, Rev. Mod. Phys. 82, 1489 (2010).

[28] R. Butté and N. Grandjean, Semicond. Sci. Tech. 26, 014030 (2011).
[29] S. Kéna-Cohen and S.R. Forrest, Nat. Photonics 4, 371 (2010)

[30] T. McGarvey, A. Conjusteau and H. Mabuchi, Optics Express 1410449 (2006).

[31] M. Koch, C. Sames, M. Balbach, H. Chibani, A. Kubanek, K. Murr, T. Wilk, and G. Rempe, Phys. Rev. Lett. 107, 023601 (2011).

[32] R. Gehr, J. Volz, G. Dubois, T. Steinmetz, Y. Colombe, B.L. Lev, R. Long, J. Estéve, and J. Reichel, Phys. Rev. Lett. 104, 203602 (2010).

[33] D. Hunger, T. Steinmetz, Y. Colombe, C. Deutsch, T. W. Hänsch, and J. Reichel, New J. Physics 12, 065038 (2010).

[34] Very recently, it has been discovered that the FMO subunit has 8 pigments. However, this should not affect the analysis we propose here for a generic light-harvesting system.

[35] M. Cho, H.M. Vaswani, T. Brixner, J. Stenger, and G.R. Fleming, J. Phys. Chem. B 109, 10542 (2005).

[36] J. Adolphs and T. Renger, Biophys. J. 91, 2778, (2006).

[37] H. Haken and G. Strobl, Z. Phys. 262, 135 (1973).

[38] A. Ishizaki and G.R. Fleming, Proc. Natl Acad. Sci. USA 106, 7255 (2009).

[39] P. Rebentrost, R. Chakraborty, A. Aspuru-Guzik, J. Chem. Phys. 131, 184102 (2009).

[40] J. Prior, A.W. Chin, S.F. Huelga, and M.B. Plenio, Phys. Rev. Lett. 105, 050404 (2010).

[41] M. Thorwart, J. Eckel, J.H. Reina, P. Nalbach, and S. Weiss, Chem. Phys. Lett. 478, 234 (2009).

[42] D. Hayes, G. Panitchayangkoon, K.A. Fransted, J.R. Caram, J. Wen, K.F. Freed, and G.S. Engel, New J. Phys. 12, 065042 (2010).

[43] L. Mandel and E. Wolf, Optical Coherence and Quantum Optics (Cambridge University Press, Cambridge, 1995).

[44] M.B. Plenio, Phys. Rev. Lett. 95, 090503 (2005). 


\section{Supplementary Information}

\section{THE MODEL}

In the site basis, the Hamiltonian of the FMO pigmentprotein complex, $H_{s}$ (in the main text), is given by

$$
\left(\begin{array}{rrrrrrr}
215 & -119.0 & 6.8 & -7.2 & 8.6 & -18.1 & -14.9 \\
-119.0 & 305 & 36.9 & 9.3 & 2.1 & 16.3 & 6.9 \\
6.8 & 36.9 & 0 & -69.8 & -1.5 & -11.5 & 3.7 \\
-7.2 & 9.3 & -69.8 & 200 & -78.3 & -21.3 & -75.9 \\
8.6 & 2.1 & -1.5 & -78.3 & 425 & 110.3 & -6.4 \\
-18.1 & 16.3 & -11.5 & -21.3 & 110.3 & 315 & 43.0 \\
-14.9 & 6.9 & 3.7 & -75.9 & -6.4 & 43.0 & 265
\end{array}\right)
$$

where the diagonal elements are the site energies - shifted from the base line $\omega_{\text {base }}=12195 \mathrm{~cm}^{-1}$ corresponding to a wavelength of $\cong 820 \mathrm{~nm}$ - while the off-diagonal elements are the inter-site coupling rates (all numbers are given in units of $\left.\mathrm{cm}^{-1}=1.988865 \cdot 10^{-23} \mathrm{Nm}=1.241410^{-4} \mathrm{eV}\right)$. The off-diagonal terms of the Hamiltonian were calculated in a dipole-dipole approximation and the site energies were taken from the Poisson-Boltzmann quantum chemistry model of Ref. [1]. The intensities of the electronic transitions in the computed absorption spectrum (top inset in Fig. 1 of the main text) are proportional to $\left|\mu_{z}\right|^{2}$, and the spectrum is averaged over different spatial orientations of the FMO complex. The dissipation and dephasing caused by the surrounding environment can be described by the following local Lindblad terms:

$$
\begin{gathered}
\mathcal{L}_{\text {diss }}^{S}(\hat{\rho})=\sum_{j=1}^{7} \Gamma_{j} / 2\left[-\left\{\hat{\sigma}_{j}^{+} \hat{\sigma}_{j}^{-}, \hat{\rho}\right\}+2 \hat{\sigma}_{j}^{-} \hat{\rho} \hat{\sigma}_{j}^{+}\right], \\
\mathcal{L}_{\text {deph }}^{S}(\hat{\rho})=\sum_{j=1}^{7} \gamma_{j} / 2\left[-\left\{\hat{\sigma}_{j}^{+} \hat{\sigma}_{j}^{-}, \hat{\rho}\right\}+2 \hat{\sigma}_{j}^{+} \hat{\sigma}_{j}^{-} \hat{\rho} \hat{\sigma}_{j}^{+} \hat{\sigma}_{j}^{-}\right],
\end{gathered}
$$

with $\Gamma_{j}$ and $\gamma_{j}$ being the dissipative and dephasing rates at the site $j$, respectively, $\hat{\sigma}_{j}^{+}\left(\hat{\sigma}_{j}^{-}\right)$being the raising (lowering) operators for site $j$. In the following, we choose for simplicity uniform dephasing rates, i.e. equal $\gamma_{j}$ and so simply labeled as $\gamma$. Moreover, here we neglect the presence of dissipation inside the FMO complex, because the 1 ns excitation lifetime [2] is much longer than the time scale we look at. Let us point out that some dissipation is also present indirectly through the interaction with the cavity which has a damping channel, described by the Lindbladian term:

$$
\mathcal{L}_{\text {diss }}^{C}(\hat{\rho})=\Gamma_{C} / 2\left(-\left\{\hat{a}^{\dagger} \hat{a}, \hat{\rho}\right\}+2 \hat{a} \hat{\rho} \hat{a}^{\dagger}\right),
$$

where $\Gamma_{C}$ being the damping rate of the cavity modes due to some dissipative processes, i.e. $\Gamma_{C}=\frac{\omega_{C}}{Q}$ with $Q$ being the quality factor of the cavity. For a self-consistent model we use the same transition dipoles to construct interactions between BChl molecules in the FMO Hamiltonian and also for the coupling with the cavity and external fields. The positions and the relative directions of the dipoles are extracted from the structure of FMO complex [3]. The positions $R_{i}$ of the 7 sites (BChl), taken as a middle point between 4 Nitrogen atoms in each BChl, are

$$
\left[R_{i}\right]=\left(\begin{array}{lll}
53.08 & 58.26 & 20.64 \\
56.04 & 54.79 & 32.40 \\
49.57 & 44.77 & 45.39 \\
38.81 & 42.25 & 43.06 \\
34.15 & 47.78 & 31.26 \\
41.44 & 47.82 & 22.61 \\
47.53 & 43.95 & 33.22
\end{array}\right)
$$

The directions the three components (in some reference frame) of the 7 induced-transition dipoles $\mu_{i}$ are

$$
\frac{\left[\mu_{i}\right]}{\mu_{0}}=\left(\begin{array}{ccc}
-0.026 & 0.286 & -0.958 \\
-0.752 & 0.601 & -0.271 \\
-0.935 & 0.061 & 0.349 \\
-0.001 & 0.393 & -0.919 \\
-0.739 & 0.672 & 0.048 \\
0.859 & 0.371 & -0.353 \\
0.176 & -0.042 & -0.983
\end{array}\right)
$$

where the absolute values of the transition dipoles are taken as a phenomenological parameter $\mu_{0}=6 \mathrm{D}$, which accounts for effects of screening and induced charges [1]. The exciton energies corresponding to the Hamiltonian (5) are $E_{1-7}=$ $[-31.0,129.4,148.7,254.6,310.1,394.5,518.6] \quad \mathrm{cm}^{-1}$ and the absolute values of the electronic transition dipoles of the FMO chromophores are $\left|\mu_{1-7}\right|=$ $[6.4,13.8,3.7,11.3,7.2,4.0,5.6] \mathrm{D}$.

Finally, we assume a continues-wave laser pumping exciton transitions in the LHC. The laser field is coupled to the cavity modes through the trapped bio-sample only. The value of the external (pump) field can be estimated as

$$
E=\sqrt{\frac{2 I}{c \varepsilon_{0}}},
$$

where $I$ is the laser field intensity, $c$ is the speed of light, and $\varepsilon_{0}$ is the vacuum permittivity. For example, the intensity of a CW laser field used in Raman spectroscopy is about $5 \mathrm{~mW}$. In these experiments the laser beam is focused on a spot of a size of $5 \mu \mathrm{m}^{2}$. This corresponds to $I=100 \mathrm{~kW} / \mathrm{cm}^{2}$ or the field $E=27 \cdot 10^{5} \mathrm{~V} / \mathrm{m}=0.45 \mathrm{~cm}^{-1} / D$. This value is much smaller than the peak field value used in femtosecond pulse lasers. However, a $\mathrm{CW}$ would result in a heat accumulation, thus stronger fields could damage a sample.

\section{EXTRACTING ADDITIONAL INFORMATION}

In this section we investigate other important features of the LHC dynamics that we can extract measuring statistical prop- 

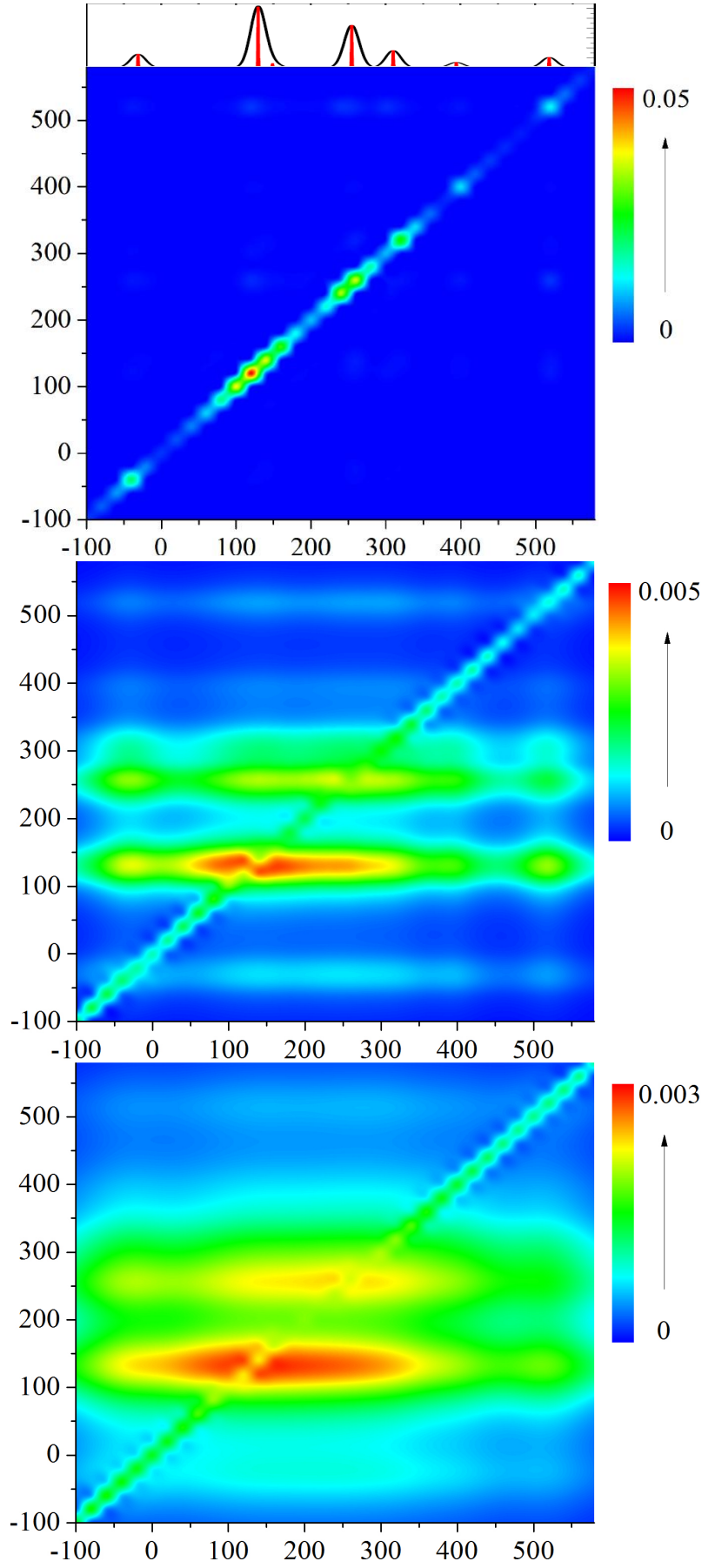

FIG. 7: Stationary mean photon number of the cavity mode as a function of the cavity $\left(Q=10^{4}\right)$ resonance frequency $\omega_{c}$ (vertical axis) and the pump laser frequency $\omega_{l}$ (horizontal), both in units of $\mathrm{cm}^{-1}$, but shifted by $12195 \mathrm{~cm}^{-1} \sim 820 \mathrm{~nm}$, for dephasing rates $\gamma=1,50,100 \mathrm{~cm}^{-1}$ (from top to bottom).

erties of the outcoming light (probe) mode. The stationary mean photon number of the outcoming light mode is shown for different values of dephasing rate $\gamma$ (in Fig. 77), also for a lower-quality $\left(Q=10^{3}\right)$ cavity (in Fig. 9 ), and for a weaker (state-of-the-art) cavity coupling rate (in Fig. 10) according to

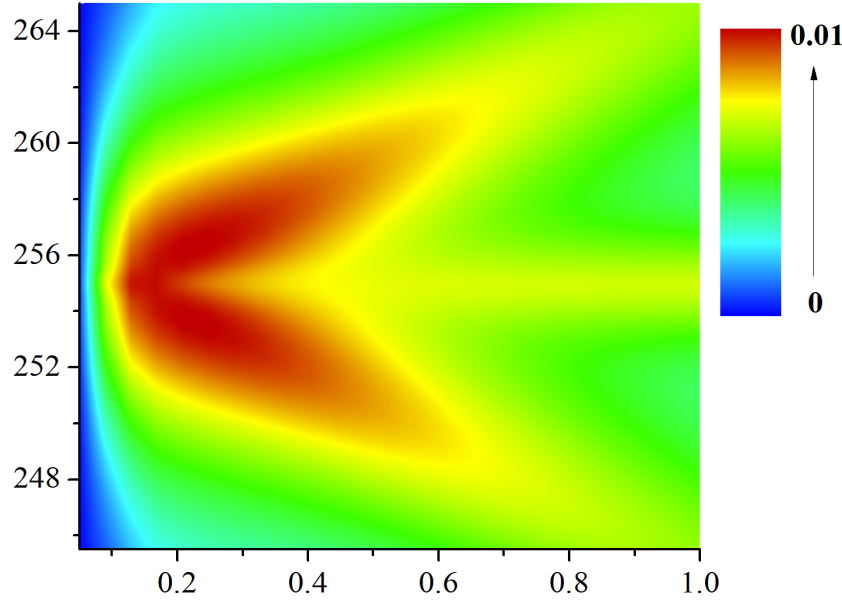

FIG. 8: Average photon number of the stationary cavity mode as a function of the laser field coupling (horizontal axis) and the cavity $\left(Q=10^{4}\right)$ resonance frequency $\omega_{c}$ (vertical) - in units $\mathrm{cm}^{-1}$ - for pump laser frequency $\omega_{l}=-\omega_{c}+510 \mathrm{~cm}^{-1}$, which corresponds to an cross-diagonal cut of a diagonal peak (red box in Fig. 2 of the main manuscript).

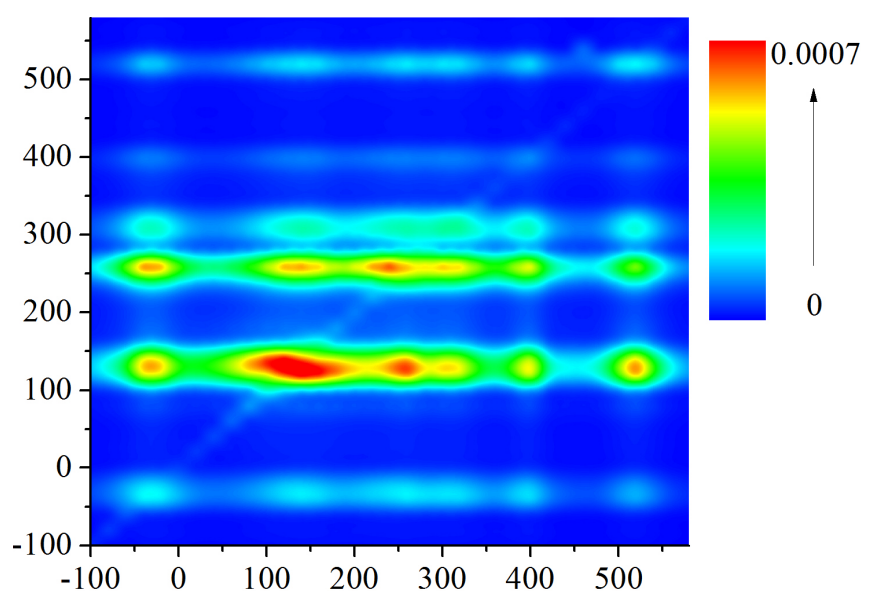

FIG. 9: Stationary mean photon number of the cavity mode as a function of the cavity $\left(Q=10^{3}\right)$ resonance frequency $\omega_{c}$ (vertical axis) and the pump laser frequency $\omega_{l}$ (horizontal), both in units of $\mathrm{cm}^{-1}$ - shifted by $12195 \mathrm{~cm}^{-1}$ - for dephasing rate $\gamma=10 \mathrm{~cm}^{-1}$.

the present stage of technological advances. The behaviour of the anti-crossing effect withing a diagonal peak as a function of the laser field coupling can be observed in Fig. 8 .

\section{Inhomogeneous broadening: static disorder}

Here, we show the effect of static disorder, i.e. disorder in the site energies, on the mean photon number 2D spectra, averaged over full random orientation disorder as well (as done in the rest of the manuscript) - see Fig. 11 for a disorder of 50 $\mathrm{cm}^{-1}$. As observed in 2D spectroscopy, it lead to the presence of inhomogeneous broadening for the diagonal peaks. 


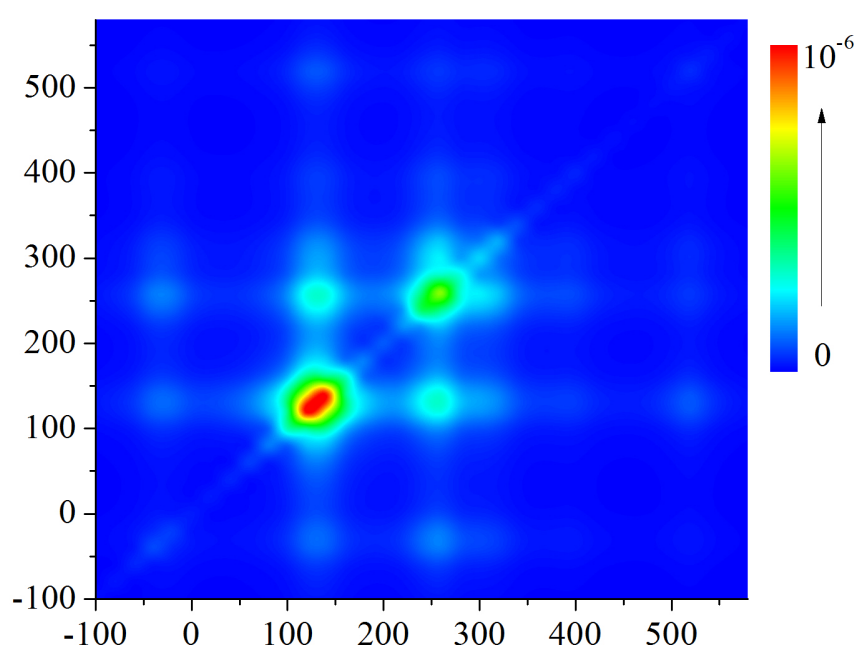

FIG. 10: Stationary mean photon number of the cavity mode as a function of the cavity $\left(Q=10^{4}\right)$ resonance frequency $\omega_{c}$ (vertical axis) and the pump laser frequency $\omega_{l}$ (horizontal), both in units of $\mathrm{cm}^{-1}$ - shifted by $12195 \mathrm{~cm}^{-1}$ - in the case of dephasing rate $\gamma=$ $50 \mathrm{~cm}^{-1}$, weaker pump field $E=0.01 \mathrm{~cm}^{-1} / D$, and weaker cavity coupling rate $g=0.01 \mathrm{~cm}^{-1} / D$.

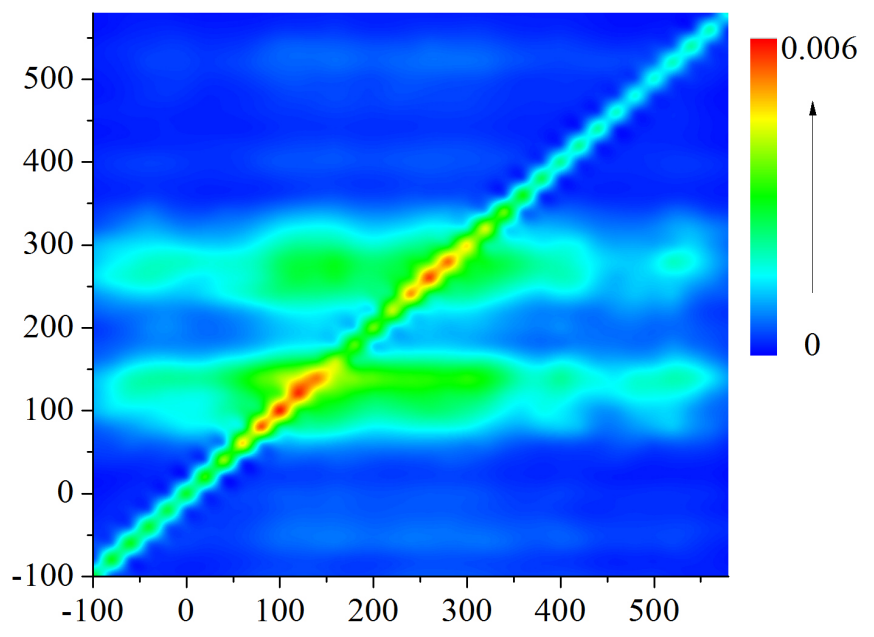

FIG. 11: Stationary mean photon number of the cavity mode as a function of the cavity $\left(Q=10^{4}\right)$ resonance frequency $\omega_{c}$ (vertical axis) and the pump laser frequency $\omega_{l}$ (horizontal), both in units of $\mathrm{cm}^{-1}$ - shifted by $12195 \mathrm{~cm}^{-1}$ - in presence of an inhomogeneous broadening, induced by a static disorder in the site energies, set to 50 $\mathrm{cm}^{-1}$, in the case of dephasing rate $\gamma=10 \mathrm{~cm}^{-1}$.

\section{Second-order correlation function}

Here, we analyze the time evolution of the second-order coherence function at time delay zero $g^{(2)}(0)$ of the stationary cavity mode (defined in the main text) for diagonal and offdiagonal peaks - see Fig. 12. Although the time scale, we consider here, is not feasible to be investigated with the current photon detector technology, however it could be in a nearfuture or even nowadays with other natural or artificial lightharvesting systems where this behavior takes place in a longer

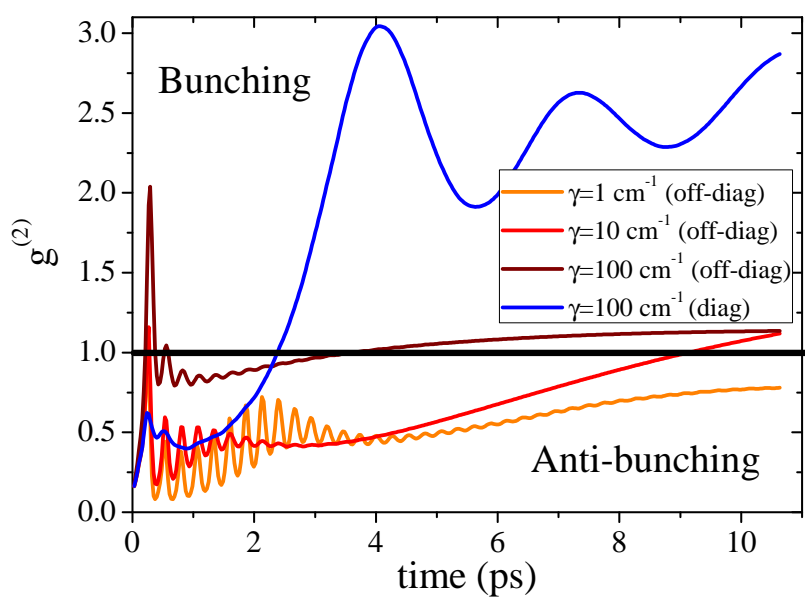

FIG. 12: Second-order coherence function at time delay zero $g^{(2)}(0)$ of the cavity $\left(Q=10^{4}\right)$ mode versus time (ps) for different values of dephasing rates and for diagonal and off-diagonal peaks, in the case of dephasing rate $\gamma=10 \mathrm{~cm}^{-1}$.

time due to different system parameters. Furthermore, in Figs. 13 we show a full-range contour-plot for the second-order coherence function at time delay zero $g^{(2)}(0)$ of the stationary cavity mode as a function of the cavity and pump frequencies, for different cavity parameters. We observe observe a formation of a quantum photon state $\left(g^{(2)}(0)<1\right)$ almost everywhere, except along the diagonal line where coherent and thermal states $\left(g^{(2)}(0) \geq 1\right)$ can appear.

\section{TRAPPING LHCS INSIDE THE CAVITY}

In our analysis it is important to trap the biological sample inside the cavity. Although several techniques are know in this respect, in this section we show, by a simple and elementary argument, the feasibility of such process by using an external electric field. In particular, let us apply an additional constant electric field $E_{0}$, polarized along the axis $\vec{e}$ with carrier frequency $\omega_{l}$, interacting with the FMO complex as the pump laser field. The corresponding interaction energy is

$$
\Delta=\sum_{i=1}^{7} \frac{\left|\vec{\mu}_{i} \cdot \vec{e} E_{0}\right|^{2}}{\omega_{l}-\omega_{i}} .
$$

and shows a minimum by varying the orientation of the polarization axis $\vec{e}$ in terms of the two angles $\theta$ and $\phi$ with respect the site- 1 dipole moment, i.e., for $\theta \sim 1.75$ and $\phi \sim 2$. In particular, we consider a spatial profile for the electric field given by a Gaussian function peaked in the center of the cavity as $E_{0}(x)=\bar{E}_{0} e^{-x^{2} /(2 \lambda)}$, where $\lambda=800 \mathrm{~nm}$. In Fig. 14, we show the trapping potential $\Delta(x)$ and the corresponding trapping force as a function of the position $x$, in the case of $\theta \sim 1.75, \phi \sim 2, \omega_{l}=-5000 \mathrm{~cm}^{-1}$, and $\bar{E}_{0}=5 \mathrm{D}^{-1} \mathrm{~cm}^{-1} \sim 30 \cdot 10^{6} \mathrm{~J} /(\mathrm{C} \mathrm{m})$. The trapping force is about 20 times larger than the gravitational force to which a single FMO is subjected, assuming that it has a mass 

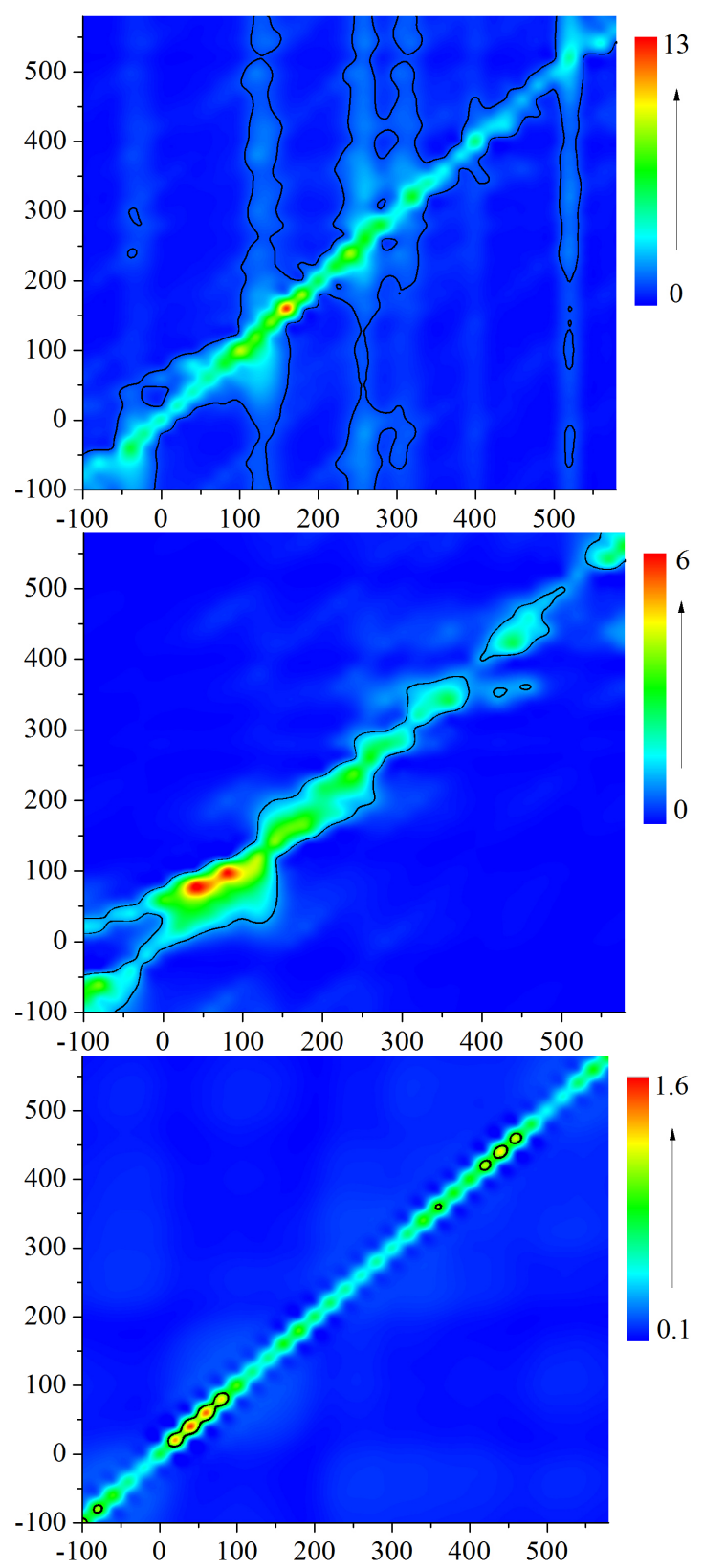

FIG. 13: Second-order coherence function at time delay zero $g^{(2)}(0)$ of the stationary cavity $\left(g=0.1 \mathrm{~cm}^{-1} / D\right.$ and $Q=10^{4}$ (Top) and $Q=10^{3}$ (Middle); Bottom: $g=0.01 \mathrm{~cm}^{-1} / D, E=$ $0.01 \mathrm{~cm}^{-1} / D$, and $Q=10^{4}$ ) as a function of the cavity resonance frequency $\omega_{c}$ (vertical axis) and the pump laser frequency $\omega_{l}$ (horizontal), both in units of $\mathrm{cm}^{-1}$ - shifted by $12195 \mathrm{~cm}^{-1}$ - for dephasing rate $\gamma=10 \mathrm{~cm}^{-1}$. The black lines refer to $g^{(2)}(0)=1$.

of around $80 \mathrm{kDa} \sim 1510^{-23} \mathrm{Kg}$ (including the protein scaffolding) [4]. In this regime, the amount of electronic excitation in the FMO complex is very small - see Inset in Fig. 14. Moreover, we have found that the trapping force can be even several order of magnitudes larger than the gravitational one, allowing a very tiny amount of excitation in the FMO system induced by the presence of the laser irradiation. Therefore, following this simple argument, it seems that it is possible to

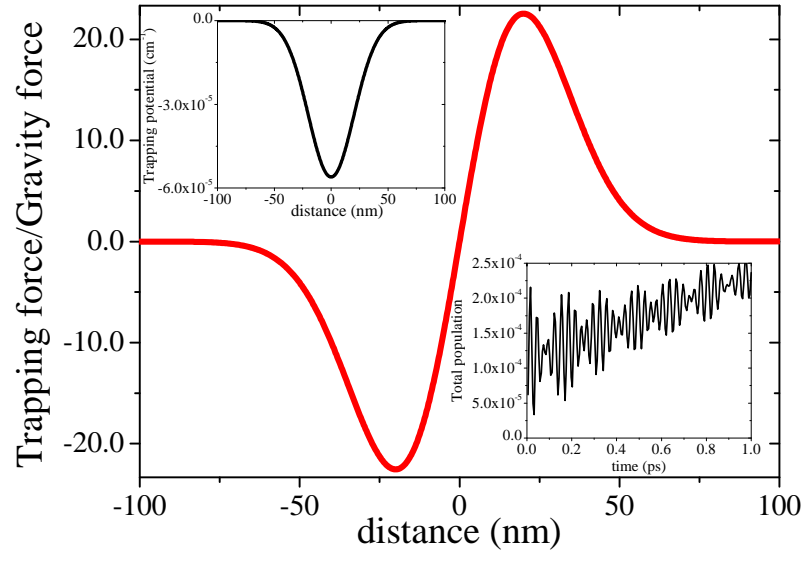

FIG. 14: Trapping force (in units of gravity force) vs. position (nm) for a single FMO complex, when subject to a constant laser field with a spatial Gaussian profile peaked in the center of the cavity, $E_{0}(x)=$ $\bar{E}_{0} e^{-x^{2} /(2 \lambda)}$ with $\bar{E}_{0}=5 D^{-1} \mathrm{~cm}^{-1} \sim 30 \cdot 10^{6} \mathrm{~J} /(\mathrm{C} \mathrm{m})$, carrier frequency set to $\omega_{l}=-5000 \mathrm{~cm}^{-1}$ and polarization orientation given by $\theta \sim 1.75, \phi \sim 2$. Top inset: trapping potential (in $\mathrm{cm}^{-1}$ ) as a function of the position (in $\mathrm{nm}$ ). Bottom Inset: in the presence of such laser irradiation (even applied for about $1 \mathrm{ps}$ ), the amount of total excitation in the FMO system is essentially vanishing, i.e. smaller than $3 \cdot 10^{-4}$.

trap a sample of FMO complexes inside the cavity, without perturbing the system, i.e. leaving it in its ground state, and then experimentally apply a driving laser field as described above.

\section{ENTANGLING BIO-SAMPLES IN SEPARATE CAVITIES}

Finally, we propose a possible scheme (in Fig. 15 for a probabilistic creation of quantum correlations, i.e. entanglement, between two FMO samples confined in spatially separate cavities, following Ref. [5] where such protocol was introduced for trapped ions. In other terms, after applying the pump laser fields to the two separate samples, the outcoming photons from the cavities are mixed on a 50/50 beam-splitter and one applies a projection of the two-photon state into a Bell state of the form $1 / \sqrt{2}(\mid$ click, no click $\rangle+\mid$ no click, click $\rangle)$. Then, after the projection, the composite system of the two LHC samples has a chance to be left in an entangled state with some finite probability. Therefore, we measure the entanglement (by log-negativity) between the two samples, after the projection for the two-cavity mode into the Bell state. This quantity is shown in Fig. 16. We find that one can probabilistically entangle the two biological samples (entangled polaritons) with a non-vanishing probability. This scheme may, for instance, allow us to extract more information on the quantum performance of these biological molecules, and perhaps pave the way for new tests about the foundation of quantum mechanics in terms of biological systems where quantum features seem to play an important role. 


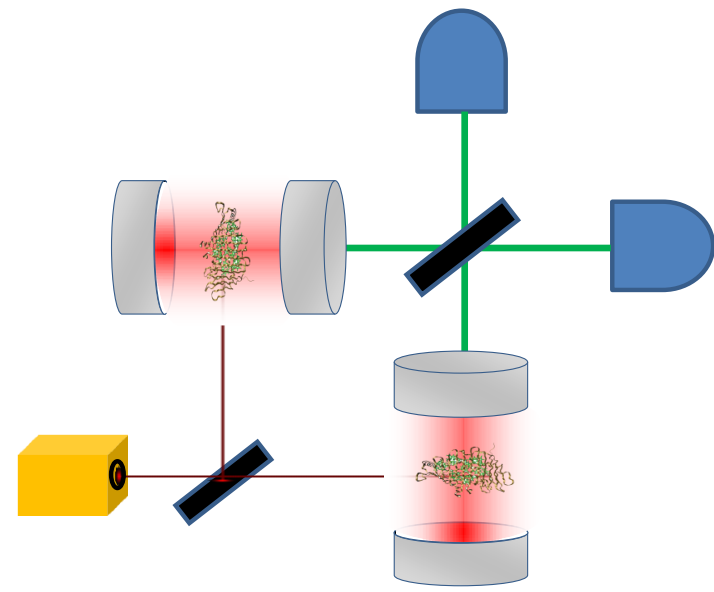

FIG. 15: We consider a setup of two equal spatially separate optical cavities in which two LHC samples are confined, while subjected to two similar pump laser fields. Two auxiliary photodectors are also introduced to detect photons, leaking out of the cavities, after being mixed on a $50 / 50$ beam-splitter.

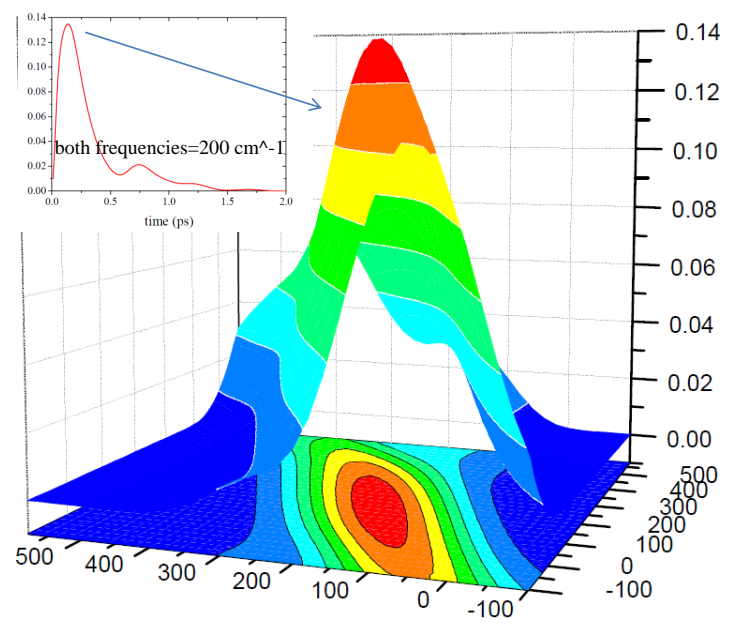

FIG. 16: Amount of quantum correlations (measured by logarithmic negativity) between the two FMO samples trapped in two spatially separate cavities, after $\sim 140 \mathrm{fs}$, in the case of dephasing rate $\gamma=10 \mathrm{~cm}^{-1}$. Inset: Entanglement vs. time (ps) for $\omega_{c}=\omega_{l}=$ $200 \mathrm{~cm}^{-1}$.
[1] J. Adolphs, F. Müh, M. El-Amine Madjet and Th. Renger, Photosynth. Res. 95, 197 (2008).

[2] J. Adolphs and T. Renger, Biophys. J. 91, 2778, (2006).

[3] Structure from PDB: 3EOJ; D.E. Tronrud. J. Wen, L. Gay, and R.E. Blankenship, Photosynth. Res. 100, 79-87 (2009).

[4] M.T.W. Milder, B. Brüggemann, v.R. Grondelle, J.L. Herek, Photosynth. Res. 194, 257-274 (2010).

[5] D.E. Browne, M.B. Plenio, and S.F. Huelga, Phys. Rev. Lett. 91, 067901 (2003). 